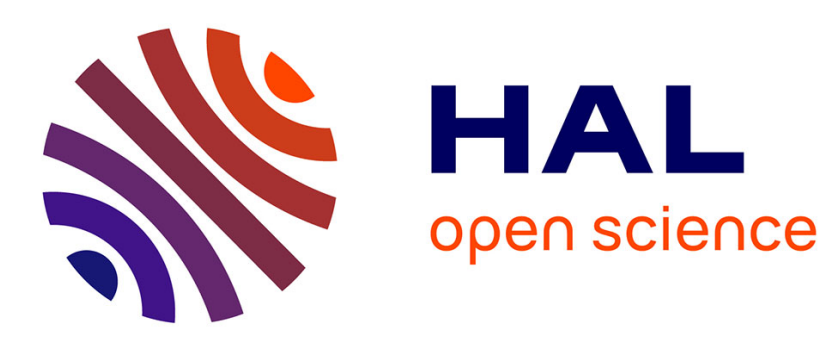

\title{
Separating Two Species of Microalgae in Photobioreactors in Minimal Time
}

\author{
Walid Djema, Olivier Bernard, Laetitia Giraldi
}

\section{To cite this version:}

Walid Djema, Olivier Bernard, Laetitia Giraldi. Separating Two Species of Microalgae in Photobioreactors in Minimal Time. Journal of Process Control, 2020, 87, pp.120-129. 10.1016/j.jprocont.2020.01.003 . hal-03148717

\section{HAL Id: hal-03148717 https://hal.inria.fr/hal-03148717}

Submitted on 22 Feb 2021

HAL is a multi-disciplinary open access archive for the deposit and dissemination of scientific research documents, whether they are published or not. The documents may come from teaching and research institutions in France or abroad, or from public or private research centers.
L'archive ouverte pluridisciplinaire HAL, est destinée au dépôt et à la diffusion de documents scientifiques de niveau recherche, publiés ou non, émanant des établissements d'enseignement et de recherche français ou étrangers, des laboratoires publics ou privés. 


\title{
Separating Two Species of Microalgae in Photobioreactors in Minimal Time
}

\author{
Walid Djema ${ }^{\mathrm{a}, *}$, Olivier Bernard ${ }^{\mathrm{a}}$, Laetitia Giraldi ${ }^{\mathrm{a}}$ \\ ${ }^{a}$ University Côte d'Azur (UCA), Inria Sophia Antipolis, France
}

\begin{abstract}
This paper proposes a strategy to separate two strains of microalgae in minimal time. The control is the dilution rate of the continuous photobioreactor. The microalgae dynamics is described by the Droop's model, taking into account the internal quota storage of the cells. Using Pontryagin's principle, we develop a dilution-based control strategy that leads to the most efficient species separation in minimal time. A numerical optimal synthesis -based on direct methods- is performed throughout the paper, in order to determine the structure of the optimal feedback-control law, which is bang-singular. Our numerical study reveals that singular arcs play a key role in the optimization problem since they allow the optimal solution to be close to an associated static optimal control problem. A resulting turnpike-like behavior, which characterizes the optimal solution, is highlighted throughout this work.
\end{abstract}

Keywords: Optimal control, Nonlinear, Pontryagin, Microalgae, Chemostat, Photobioreactor.

\section{Introduction}

For a single limiting resource, the competitive exclusion principle (CEP) ([19]) in a continuous reactor states that one of the species wins the competition to the detriment of the others. This concept has been widely used in ecology, but more rarely applied in biotechnology, with the objective of selecting a strain with enhanced productivity of some interesting products. It has recently been shown ([20], Chap. 12) that an efficient microorganism selection can be achieved through a competition process taking place in continuous cultures (see also [9] and the references therein).

The chemostat is a continuous reactor in which the principle of competition occurs either between different species initially coexisting, or even between different strains of the same species. The basic modeling framework given by the Monod's model is the most used to represent microorganisms growing in the chemostat $([22,23])$. This model first supported the CEP explaining the competition outcome in the chemostat (see, e.g., [25]). More specifically, the CEP predicts that if several species are introduced in the chemostat, the one that requires

*Corresponding author.

Email addresses: walid.djema@inria.fr (Walid Djema), olivier.bernard@inria.fr (Olivier Bernard), laetitia.giraldi@inria.fr (Laetitia Giraldi) 
the less nutrient to sustain a growth rate equal to the dilution rate will win the competition, while the other species will vanish out asymptotically $([18,25])$. Many works have focused on controlling the chemostat system in order to select other species for winning the competition, according to more attractive and practical criteria ([21], see also [15]). More recently, some approaches based on optimal control theory have been applied to Monod's model, in order to drive and accelerate the CEP, leading to species selection in finite time ([1, 2]). The application of these strategies to microalgae is not straightforward since the Monod's model is not a convincing approximation (see, e.g., [4, 5]). Indeed, microalgae have the ability to store internally the limiting nutrient before using it for growth. These storage mechanisms cannot be captured by the classical Monod's model, and a more suitable framework for microalgae growth is provided by the so-called Droop's model $([12-14,18,25])$. In fact, Droop's model includes a new dynamics where an internal nutrient storage is introduced, so that only nutrients internal to the cell are available for cell growth (see, e.g., [10]). The Droop's model is also known as the variable yield model, as it no longer assumes a constant ratio between cell growth and nutrient uptake rate ([25]). Indeed, the growth rate in Droop's dynamics is empirically determined, only from the additional state variable quantifying the nutrient quota in the cell.

A preliminary study using a simplified version of Droop's model, corresponding to the case where the set of possible initial conditions are confined into a positively invariant set, has been performed in [11]. This assumption -that we no longer consider in this paper- allowed to reduce the dimension of the studied model. Notice also that an equivalent hypothesis is also usually considered in the simpler Monod's model (see, e.g., [1]). But however it turns that such a simplification limits the scope of the previous study made on Droop's model in [11]. Indeed, we show in the rest of the paper that the time required to separate the species is substantially similar to the one required by the trajectories to reach the positively invariant set of interest. Finally, it is worth mentioning that introducing the quota dynamics and abandoning the restriction on the initial conditions increase the dimension of the studied model, and thus making its mathematical analysis more difficult. So, this work is devoted to the analysis of a competition model involving two species described with the full Droop kinetics. This can be seen as a generalization of the approach of $[1,11]$, with a more complex class of systems involving additional states (i.e., the internal quota of each species and the total substrate concentration). In particular, geometrical arguments used for species selection in Monod's model are difficult to apply in the Droop's case, due to the state's dimension.

The paper is organized as follows. The Droop's model involving two species is introduced in Sect. 2. The targeted minimal-time optimal control problem (OCP) is stated in Sect. 3. This is the natural OCP that aims to maximize the ratio between two biomass concentrations in minimal time. Besides, we derive a suitable associated OCP over a fixed time-window in Sect. 4, and then from its static version a useful optimal steady-state is defined. Next, in Sect. 5, Pontryagin's Maximum Principle (PMP) is applied on the minimal-time OCP in order to determine the optimal control for the dilution rate. A numerical optimal synthesis is then carried out in Sect. 6, based on direct methods to solve the minimal-time OCP. In particular, we illustrate how the optimal solution of the minimal-time OCP approaches 
the useful optimal steady-state, a behavior that is characterized as a turnpike feature of the optimal solution.

\section{The Droop's quota model}

Two microalgae species (or strains) of concentration $x_{1}$ and $x_{2}$ are initially growing in a chemostat with one limiting substrate $s$. Their internal nutrient quota is denoted by $q_{i}$. The total amount of nutrient stored in the cells at time $t \geq 0$ is $\sum_{i=1}^{2} q_{i}(t) x_{i}(t)$. The cell quota $q_{i}$, for $i=1,2$, is fueled by substrate uptake and is diluted with cell growth. The variable yield model -Droop's model- involving two competing species (or strains) is given by,

$$
\left\{\begin{array}{l}
\dot{s}=\left(s_{i n}-s\right) D-\sum_{i=1}^{2} \rho_{i}(s) x_{i}, \\
\dot{q}_{i}=\rho_{i}(s)-\mu_{i}\left(q_{i}\right) q_{i}, \\
\dot{x}_{i}=\left[\mu_{i}\left(q_{i}\right)-D\right] x_{i},
\end{array}\right.
$$

where $i=1,2$. The scalar variable $s$ is the total free substrate concentration, and $s_{i n}$ is the constant input concentration of the substrate in the photobioreactor. The dilution rate, denoted by $D$, is the control function in the chemostat. This is a bounded nonnegative control s.t., for all $t \geq 0, D(t) \in\left[0, D_{\max }\right]$, where the upper bound $D_{\max }$ corresponds to the maximum capacity of the feeding pumps. Next, $\rho_{i}$ is a real-valued function quantifying the uptake rate of free nutrient $s$; while $\mu_{i}$ is a real-valued function quantifying the growth rate of the $i$-th species [3]. The functions $\rho_{i}$ and $\mu_{i}$ are nonnegative and increasing bounded functions, over appropriate definition sets, s.t.,

$$
0 \leq \rho_{i}(s) \leq \rho_{m i}, \quad 0 \leq \mu_{i}\left(q_{i}\right) \leq \mu_{m i}
$$

where $\rho_{m i}$ and $\mu_{m i}$ are positive constants. Typically in the Droop's model, the uptake rate $\rho_{i}$ is expressed in terms of Michaelis-Menten kinetics,

$$
\rho_{i}(s)=\frac{\rho_{m i} s}{K_{s i}+s}, \text { for all } s \geq 0,
$$

where $K_{s i}$ is a positive constant of the $i$-th species. For the growth functions, we consider that there exists a minimum threshold $k_{q i}>0$, for each species, under which growth cannot occur, and we consider the growth rates in the Droop form,

$$
\mu_{i}\left(q_{i}\right)=\mu_{i \infty}\left(1-\frac{k_{q i}}{q_{i}}\right), \quad q_{i} \geq k_{q i}
$$

In fact, let us quote that for all $t \geq 0, k_{q i} \leq q_{i}(t) \leq q_{m i}$, where $q_{m i}$ is the maximum internal storage rate, and $\mu_{m i}=\mu_{i}\left(q_{m i}\right)$ corresponds to the maximum growth rate for the $i$-th species (see, e.g., [3]), and finally, $\mu_{i \infty}=\frac{q_{m i}}{q_{m i}-k_{q i}} \mu_{m i}$. In addition, to be consistent with inequalities (2), we have, $\rho_{m i}=\mu_{i}\left(q_{m i}\right) q_{m i}$, where, $\mu_{i}\left(q_{m i}\right)=\frac{\rho_{m i}}{q_{m i}}=\mu_{m i}$. 
Clearly, the studied system (1) is positive, i.e., for strictly positive initial conditions the trajectories remain positive for all future time. The total mass of limiting element in the chemostat system is given by, $z=s+q_{1} x_{1}+x_{2} q_{2}$. In addition, in quota models, the set,

$$
\mathcal{F}=\left\{X:=\left(s, q_{1}, q_{2}, x_{1}, x_{2}\right) \in \mathcal{S} \mid k_{q i} \leq q_{i} \leq q_{m i}, \quad z=s_{i n}\right\},
$$

where, $\mathcal{S}=\mathbb{R}_{+}^{*} \times\left[k_{q 1}, q_{m 1}\right] \times\left[k_{q 2}, q_{m 2}\right] \times \mathbb{R}_{+}^{*} \times \mathbb{R}_{+}^{*}$, is positively invariant and attractive for system (1), i.e., the total mass remains constant for all future time, $z=s_{i n}$, as long as the initial conditions are within $\mathcal{F}$. Consequently, considering that the initial conditions associated to system (1) belong to the set $\mathcal{F}$ allows to reduce the dimension of system (1), since $s=s_{\text {in }}-q_{1} x_{1}-q_{2} x_{2}$ for all future time. This hypothesis has been assumed in our previous work ([11]) but no longer in the present one. As we will show in Sect. 6, the time required to converge to the target set is substantially similar to the time to reach the positively invariant set $\mathcal{F}$, meaning that the assumption in [11] is to a certain extent too conservative and jeopardizes the interest of the optimal control strategy.

\section{Statement of the minimal-time Optimal Control Problem (OCP)}

Now, let us express the natural Optimal Control Problem (OCP) so that species of $x_{1}$ dominates in minimum time. We define the target of interest $\mathcal{T}_{\epsilon}$ as follows,

$$
\mathcal{T}_{\epsilon}=\left\{X:=\left(s, q_{1}, q_{2}, x_{1}, x_{2}\right) \in \mathcal{S} \mid x_{2}<\epsilon x_{1}\right\},
$$

where $X$ satisfies system (1) and $\epsilon$ is a small enough positive constant defining the acceptable contamination level of species $x_{1}$ by $x_{2}$. The objective is to determine a dilution-based optimal control strategy $D$ that steers the trajectories of system (1), starting from arbitrary initial conditions $X(0) \in \mathcal{S}$, in order that $X$ reaches the target $\mathcal{T}_{\epsilon}$ in minimal time $t_{f}$. For that, we define firstly the set of admissible controls,

$$
\mathcal{D}=\left\{D:[0,+\infty] \rightarrow\left[0, D_{\max }\right] \mid D(\cdot) \in \mathcal{L}_{\text {loc }}^{\infty}\left(\mathbb{R}^{+}\right)\right\},
$$

where we recall that $D_{\max }$ is a sufficiently large strictly positive constant, and $\mathcal{D}$ is a subset of $\mathcal{L}_{\text {loc }}^{\infty}\left(\mathbb{R}^{+}\right)$, the space of locally integrable functions on every compact set on $\mathbb{R}^{+}$.

The natural OCP of interest is stated as follows. For all initial conditions belonging to $\mathcal{S}$, we are seeking an admissible control strategy $D \in \mathcal{D}$, steering the solution to the target set $\mathcal{T}_{\epsilon}$ in minimum time $t_{f}$, i.e., for a fixed $D_{\max }$ and a given contamination rate $\epsilon$, the natural OCP reads,

$$
\left\{\begin{array}{l}
\inf _{D \in \mathcal{D}} t_{f}, \quad \text { s.t. } \quad X\left(t_{f}\right) \in \mathcal{T}_{\epsilon} \\
X(\cdot) \text { is solution of }(1), \text { and } X(0) \in \mathcal{S} .
\end{array}\right.
$$

In Sect. 5, the OCP (6) is investigated using Pontryagin's theory, then its numerical solution is provided using direct optimization methods in Sect. 6. Before that we go through a slightly different representation in Sect. 4 in order to define a useful optimal steady-state that plays a key role in the optimal-control strategy solution of the OCP (6). 


\section{Insights from an associated static-OCP over a fixed time-horizon}

In this section, we firstly show that for a given $D_{\max }$ and a contamination rate $\epsilon$ in the target $\mathcal{T}_{\epsilon}$, the solution of the minimal-time OCP (6) is also an optimal solution of an associated OCP, which is formulated over a fixed time-window $\left[0, t_{f}^{*}\right]$. Here $t_{f}^{*}$ is the optimal minimal-time obtained when solving the OCP (6). Therefore, from the definition of the target set $\mathcal{T}_{\epsilon}$, it follows that,

$$
x_{2}\left(t_{f}^{*}\right)=\epsilon x_{1}\left(t_{f}^{*}\right), \text { and, } x_{1}(t) / x_{2}(t)<1 / \epsilon, \text { for all } t \in\left[0, t_{f}^{*}\right) .
$$

In particular, from (7) it is straightforward that the optimal solution of the OCP (6) maximizes the ratio $x_{1} / x_{2}$ at $t=t_{f}^{*}$, and this is the maximum over the time interval $\left[0, t_{f}^{*}\right]$. Thus, we can readily check that the optimal solution of the OCP (6), providing the optimal $t_{f}=t_{f}^{*}$, satisfies also the criterion,

$$
\sup _{D \in \mathcal{D}} \int_{0}^{t_{f}} \mu_{1}\left(q_{1}(t)\right)-\mu_{2}\left(q_{2}(t)\right) d t, \text { for } t_{f}=t_{f}^{*}
$$

To see why this holds let us note that from the Droop's dynamics (1) it follows that,

$$
\int_{0}^{t_{f}} \mu_{1}\left(q_{1}(t)\right)-\mu_{2}\left(q_{2}(t)\right) d t=\ln \left(\frac{x_{1}\left(t_{f}\right) x_{2}(0)}{x_{2}\left(t_{f}\right) x_{1}(0)}\right) .
$$

In other words, satisfying (8) leads to the maximization of the ratio $x_{1} / x_{2}$ at $t=t_{f}$, which is also equivalent to reaching $\mathcal{T}_{\epsilon}$, for a suitable $\epsilon$, in optimal time $t_{f}=t_{f}^{*}$. Now, OCP (6) reads as an optimal control problem with a cost function expressed as an integral over a fixed time of the difference between growth rates. As in [26], we define the so-called static-OCP associated with the fixed-time OCP (8). The static-OCP provides some key elements to understand the behavior of the solutions of the minimal-time OCP (6).

From (8) and Droop's dynamics (1), we define the following static-OCP,

$$
\sup _{D \in\left[0, D_{\text {max }}\right]} \mu_{1}\left(q_{1}\right)-\mu_{2}\left(q_{2}\right), \quad \text { subject to, }\left\{\begin{array}{l}
\left(s_{i n}-s\right) D-\sum_{i=1}^{2} \rho_{i}(s) x_{i}=0 \\
\rho_{i}(s)-\mu_{i}\left(q_{i}\right) q_{i}=0 \\
{\left[\mu_{i}\left(q_{i}\right)-D\right] x_{i}=0 .}
\end{array}\right.
$$

In order to determine the solution of the static-OCP (9), let us observe that for a fixed admissible dilution-rate $D^{*} \in\left[0, D_{\max }\right]$, the external substrate in the static problem (9) is fixed to $s^{*}=s\left(D^{*}\right)$, where $s^{*} \in\left[0, s_{i n}\right]$. Then, it follows that the cell-quota is fixed to $q_{i}^{*}=$ $q_{i}\left(s^{*}\right)=q_{i}\left(s\left(D^{*}\right)\right)$, where, $q_{i}^{*} \in\left[k_{q i}, q_{i m}\right]$ is the unique solution of $\mu_{i}\left(q_{i}^{*}\right) q_{i}^{*}=\rho_{i}\left(s^{*}\right)$. From $\rho_{i}$ and $\mu_{i}$ given in (3)-(4), we define the function, $\delta_{i}(a)=\rho_{i}^{-1}\left(\tilde{\mu}_{i}(a)\right)=K_{s i} \frac{a-k_{q i}}{\kappa_{i}-a}, a \in\left[0, \kappa_{i}\right)$, where, $\tilde{\mu}_{i}(a)=\mu_{i}(a) a$, and, $\kappa_{i}=\frac{\rho_{m i}}{\mu_{i \infty}}+k_{q i}$. Therefore, $s^{*}=\delta_{i}\left(q_{i}\left(s^{*}\right)\right)$, for $i=1,2$, and the cell quota is fixed to, $q_{i}\left(s^{*}\right)=\delta_{i}^{-1}\left(s^{*}\right)$, for $i=1,2$, where, $\delta_{i}^{-1}\left(s^{*}\right)=\frac{\kappa_{i} s^{*}+K_{s i} k_{q i}}{s^{*}+K_{s i}}$. Therefore, we define for any fixed value $s^{*}\left(D^{*}\right) \in\left[0, s_{i n}\right]$, and its associated $q_{i}^{*}\left(s^{*}\right)=\delta_{i}^{-1}\left(s^{*}\right) \in\left[k_{q i}, q_{i m}\right]$, the effective growth-rate of each microalgae species,

$$
\mu_{i}\left(\delta_{i}^{-1}\left(s^{*}\right)\right)=\frac{\rho_{m i} s^{*}}{\kappa_{i} s^{*}+K_{s i} k_{q i}} .
$$


Consequently, using (10) we define the difference between the effective growth-rates,

$$
\Delta\left(s^{*}\right)=\mu_{1}\left(\delta_{1}^{-1}\left(s^{*}\right)\right)-\mu_{2}\left(\delta_{2}^{-1}\left(s^{*}\right)\right), \quad s^{*}=s\left(D^{*}\right) \in\left[0, s_{i n}\right] .
$$

Thus, we have transformed, through Droop's static equations (9), the difference $\mu_{1}\left(q_{1}\right)-$ $\mu_{2}\left(q_{2}\right)$ to be maximized in the static-OCP (9), into the function $\Delta\left(s^{*}\right)$ in (11), where $s^{*}$ is a static solution belonging to $\left[0, s_{i n}\right]$. In particular, the maximization of $\mu_{1}\left(q_{1}\right)-\mu_{2}\left(q_{2}\right)$ in the static-OCP is now up to the maximization of the function $\Delta$ for some static optimal substrate $s\left(D^{*}\right)$. Regarding the expression of functions $\mu_{i}\left(\delta_{i}^{-1}(\cdot)\right), i=1,2$, the function $\Delta$ in (11) has a unique global maximum denoted by $s_{c} \in\left[0, s_{i n}\right]$, i.e., $\exists ! s_{c} \in\left[0, s_{i n}\right]$, s.t. $\Delta\left(s_{c}\right)>\Delta\left(s^{*}\right), \forall s^{*} \in\left[0, s_{i n}\right]$ and $s^{*} \neq s_{c}$, as illustrated in Sect. 6. Thus, the optimal and unique steady-state satisfying $(9)$ reads,

$$
\mathcal{E}=\left(s_{c}, q_{1 c}, q_{2 c}\right), \quad \text { s.t. }, \quad q_{i c}=\delta_{i}^{-1}\left(s_{c}\right), \quad i=1,2 .
$$

The optimal steady-state $\mathcal{E}$ has a biological meaning, since the value $s_{c}$ maximizing $\Delta$ characterizes an optimal mode that achieves largest difference between the effective growthrates of the two species. It is then expected at a first sight that in order to achieve optimal separation between the two species, the optimal trajectories of the OCP (6) should remain close to the critical point $\mathcal{E}$. Let us quote that a similar feature appears when separating two species driven by the Monod's model (see [1]). However, in the Droop's case we go further by characterizing the point $\mathcal{E}$, solution of the static-OCP (9), as a turnpike of the fixed-time problem (8), which is also reflected on the optimal solution of the minimal-time OCP (6). We recall that the turnpike property is formally demonstrated for fixed-time OCPs, describing the behavior of optimal solutions that follow over a time-interval the solution of their associated static OCP (see [26, 27]). Sect. 6 will particularly highlight the turnpike-type behavior that appears in the OCP (6).

In the next section, we apply Pontryagin's principle ([24]) on the natural minimal-time OCP (6) in order to provide necessary conditions for the optimality of the control $D$.

\section{Pontryagin Maximum Principle (PMP)}

We denote,

$$
H=H\left(s, q_{1}, q_{2}, x_{1}, x_{2}, \lambda_{q_{1}}, \lambda_{s}, \lambda_{q_{2}}, \lambda_{x_{1}}, \lambda_{x_{2}}, \lambda_{0}, D\right),
$$

the Hamiltonian of system (1) associated with the OCP stated in (6). By definition, $H$ is given by,

$$
\begin{aligned}
H= & D \Phi\left(s, x_{1}, x_{2}, \lambda_{s}, \lambda_{x_{1}}, \lambda_{x_{2}}\right)+\lambda_{0}-\left(\rho_{1}(s) x_{1}+\rho_{2}(s) x_{2}\right) \lambda_{s}+\left(\rho_{1}(s)-\mu_{1}\left(q_{1}\right) q_{1}\right) \lambda_{q_{1}} \\
& +\left(\rho_{2}(s)-\mu_{2}\left(q_{2}\right) q_{2}\right) \lambda_{q_{2}}+\mu_{1}\left(q_{1}\right) x_{1} \lambda_{x_{1}}+\mu_{2}\left(q_{2}\right) x_{2} \lambda_{x_{2}},
\end{aligned}
$$

where,

$$
\Phi\left(s, x_{1}, x_{2}, \lambda_{s}, \lambda_{x_{1}}, \lambda_{x_{2}}\right)=-\lambda_{x_{1}} x_{1}-\lambda_{x_{2}} x_{2}+\left(s_{i n}-s\right) \lambda_{s} .
$$

The co-states of $s, q_{1}, q_{2}, x_{1}$ and $x_{2}$ are governed by,

$$
\dot{\lambda}_{s}=-\frac{\partial H}{\partial s}, \quad \dot{\lambda}_{q_{1}}=-\frac{\partial H}{\partial q_{1}}, \quad \dot{\lambda}_{q_{2}}=-\frac{\partial H}{\partial q_{2}}, \quad \dot{\lambda}_{x_{1}}=-\frac{\partial H}{\partial x_{1}}, \quad \dot{\lambda}_{x_{2}}=-\frac{\partial H}{\partial x_{2}},
$$


and the state variables of system (1) satisfy,

$$
\dot{s}=\frac{\partial H}{\partial \lambda_{s}}, \quad \dot{q}_{1}=\frac{\partial H}{\partial \lambda_{q_{1}}}, \quad \dot{q}_{2}=\frac{\partial H}{\partial \lambda_{q_{2}}}, \quad \dot{x}_{1}=\frac{\partial H}{\partial \lambda_{x_{1}}}, \quad \dot{x}_{2}=\frac{\partial H}{\partial \lambda_{x_{2}}},
$$

with $X(0) \in \mathcal{S}$. Next, in Pontryagin's approach, the control $D$ satisfies the maximization condition,

$$
D(t) \in \underset{D(t) \in\left[0, D_{\max }\right]}{\operatorname{argmax}} H,
$$

for almost all $t \in\left[0, t_{f}\right]$, where $t_{f}$ is the first time the trajectories reach the target $\mathcal{T}_{\epsilon}$. It is classical to set $\lambda_{0}=-1$ in minimization problems. Now, let us express the transversality conditions corresponding to the minimal-time OCP (6). By definition, the co-state vector,

$$
\Lambda(t)=\left[\begin{array}{lllll}
\lambda_{s}(t) & \lambda_{q_{1}}(t) & \lambda_{x_{1}}(t) & \lambda_{q_{2}}(t) & \lambda_{x_{2}}(t)
\end{array}\right]^{t r}
$$

satisfies $^{1}$ at the final-time $t=t_{f}$,

$$
\Lambda\left(t_{f}\right) \in \mathcal{N}_{\mathcal{T}_{\epsilon}}\left(X\left(t_{f}\right)\right)
$$

where $X$ is solution of (1) and $\mathcal{N}_{\mathcal{T}_{\epsilon}}$ is the normal cone to the target $\mathcal{T}_{\epsilon}$ at the final-point $X\left(t_{f}\right)$. Hence, from the definition of the target $\mathcal{T}_{\epsilon},(16)$ expresses that,

$$
\Lambda^{\operatorname{tr}}\left(t_{f}\right)\left[\begin{array}{cccc}
1 & 0 & 0 & 0 \\
0 & 1 & 0 & 0 \\
0 & 0 & 1 & 0 \\
0 & 0 & 0 & 1 \\
0 & 0 & \epsilon & 0
\end{array}\right]=0 .
$$

Therefore, it follows that,

$$
\lambda_{s}\left(t_{f}\right)=\lambda_{q_{1}}\left(t_{f}\right)=\lambda_{q_{2}}\left(t_{f}\right)=0 .
$$

In addition, it follows from (17) that the vector $\left[\begin{array}{lll}\lambda_{x_{1}}\left(t_{f}\right) & \lambda_{x_{2}}\left(t_{f}\right)\end{array}\right]^{t r}$ is parallel to the vector $\left[\begin{array}{ll}\epsilon & -1\end{array}\right]^{t r}$. Therefore, we conclude that, with an abuse of notation,

$$
\Phi\left(t_{f}\right)=0 .
$$

On the other hand, since the Hamiltonian $H$ is linear with respect to the control $D$, then we deduce that the control law $D$ is given by the sign of the switching function $\Phi$, i.e.,

- $D=D_{\max } \quad$ iff $\Phi>0$.

- $D=0$ iff $\Phi<0$.

\footnotetext{
${ }^{1}$ The overscript $t r$ means the transpose of the vector/matrix.
} 
- $D=D_{s}$, when $\Phi=0$, where $D_{s} \in\left(0, D_{\max }\right)$ is the singular control.

The analytical expression of $D_{s}$ in the particular case where the initial conditions are confined to the positively invariant set $\mathcal{F}$ is given in [11]. Thus, the Pontryagin's principle provides guidelines on the form of the optimal control, which can combine bang controls (0 and/or $\left.D_{\max }\right)$ and singular $\operatorname{arcs} D_{s} \in\left(0, D_{\max }\right)$. The key point that we want to highlight here is that the condition (19) implies that the target $\mathcal{T}_{\epsilon}$ is reached with the singular control $D_{s}$. The role of the control $D$ is firstly to put the system in an operating mode separating the species in minimal time, which is close to the steady-state $\mathcal{E}$, since $s_{c}$ maximizes the function $\Delta(s)=\mu_{1}\left(\delta^{-1}(s)\right)-\mu_{2}\left(\delta^{-1}(s)\right)$, as discussed in Section 4. Then, in a second phase, the singular arc -or the singular control $D_{s}$ - steers the states $x_{i}$ to the target $\mathcal{T}_{\epsilon}$.

In the next section, we fully-characterize numerically the structure of the optimal control using a direct method, i.e., by discretizing the optimal control problem and solving a nonlinear programming problem ([6], [7]).

\section{A numerical optimal synthesis}

Using the arguments of Sect. 4 and 5, we state that the optimal control solution of OCP (6) is achieved through two steps. The first step aims at driving the system from the initial condition close to the value $s=s_{c}$ using a bang-type control. Then, the second step consists in remaining as close as possible to $s=s_{c}$, showing a turnpike-like feature, by using the singular control $D_{s} \in\left(0, D_{\max }\right)$ maximizing the Hamiltonian $H$. Thus, we state the following conjecture.

Conjecture 1. There exists a time $t_{s}>0$ such that the optimal control solution of (6) is decomposed as follows,

$$
\forall t<t_{s}, \quad D(t)=0, \quad \text { or } \quad D_{\max }
$$

and

$$
\forall t \geq t_{s}, \quad D(t)=D_{s}(t)
$$

It is worth mentioning that this bang-singular type control is similar to the one observed in Monod's model ([1]), with the notable exception that the substrate $s$ is no longer constant along the singular arc in our case. More importantly, the switching time, denoted by $t_{s}$ throughout this section, does not correspond to $s\left(t_{s}\right)=s_{c}$, as it was the case in [1].

To validate numerically these statements, we determine the optimal synthesis numerically, using the biological parameters and functions given in Table 1. These numerical values ensure the existence of an intersection point between $\mu_{1}\left(\delta_{1}^{-1}(s)\right)$ and $\mu_{2}\left(\delta_{2}^{-1}(s)\right)$ as well as the generic form of interest of the function $\Delta$ illustrated in Figure 1.

The direct method is implemented using Bocop software ${ }^{2}[8]$, where the states and the control variables of the studied system are discretized. More precisely, the dilution-rate control $D$ is discretized over $\left[0, D_{\max }\right]$ such that it is piecewise constant on the time-interval

\footnotetext{
${ }^{2}$ Optimal control solver, https://www.bocop.org/
} 
subdivision. The state dynamics need also to be discretized through a scheme of ordinary differential equations (ODE) decomposition (e.g., Euler method, Runge-Kutta, etc.). Thus, numerical direct methods transform the OCP of interest, by discretizing the states and the control, into a nonlinear programming problem (NLP) in finite dimension. We use in Bocop a variant of Lobatto methods for numerical integration of ODEs $([6,7])$. We note in particular that these methods are well adapted to stiff and difficult problems [16]. The nonlinear optimization problem is solved in Bocop using an interior point approach, with a discontinuous collocation method of Lobatto's type (a sixth order time-discretization Labatto III-C formula). The tolerance for NLP solver is tuned to $10^{-38}$.

In the numerical illustrations presented below, we point out the link between the switching instant $t_{s}$ and the dynamics of the co-state $\lambda_{s}$. Indeed, $t_{s}$ corresponds to the time at which the co-state $\lambda_{s}$ becomes zero, leading to a non-smooth trajectory, and then $\lambda_{s}$ remains zero for all $t \in\left[t_{s}, t_{f}\right]$ in order to satisfy the transversality condition when reaching the target, i.e., $\lambda_{s}\left(t_{f}\right)=0$. This behavior is illustrated in Figures 4,8 and 11.

Furthermore, we highlight that in the Monod's model the optimal trajectory of the substrate concentration $s$ coincides exactly with the constant $s_{c}$ that maximizes the function $\Delta$. The previous observation (from [1]) seems quite natural in Monod's model, however Droop's model is less trivial to interpret since the variables $q_{i}$ introduce a latency (i.e., they act as time-delays) between the absorption of $s$ and the growth of the species $x_{i}$, in a nontrivial way. Thus, the strategy achieves better performances through an optimal solution where the substrate concentration $s$ is not exactly constant. From extensive simulations, the two following cases summarize the numerical optimal synthesis, depending the initial condition $s^{0}$ of the substrate:

(1) If $s^{0}>s_{c}$, the control steers $s$ to a neighborhood of $s_{c}$ using $D=0$. Indeed, when $D=0$, we get $\dot{s}<0$ and so the $s$ variable decreases. Then, the control switches to the singular control $D_{s}$, which steers the trajectories to the target $\mathcal{T}_{\epsilon}$ in minimal time.

2 If $s^{0}<s_{c}$, the control $D=D_{\max }$ is the fastest way to steer $s$ to a neighborhood of $s_{c}$. Then, as in the previous case, the control switches to the singular control $D_{s}$ that steers the trajectories to the target $\mathcal{T}_{\epsilon}$ in minimal time. Let us observe that the dynamics of $x_{i}$ in closed loop with $D=D_{\max }$ are governed by, $\dot{x}_{i}=\left[\mu_{i}\left(q_{i}\right)-D_{\max }\right] x_{i}$. We deduce that the species concentrations $x_{i}$ converge exponentially to zero when $D_{\max }$ is sufficiently large (e.g., $D_{\max }>\mu_{m i}$, as it is usually the case when fixing $D_{\max }$ in the photobioreactor). Thus, it follows that $s$ converges to $s_{\text {in }}$ when $t \rightarrow \infty$. Therefore, for $s_{i n}$ large enough (as it is generally the case in the experiments) $s$ increases and approaches $s_{c} \in\left[0, s_{i n}\right]$ in finite time.

The behaviors outlined in (1) and (2) are highlighted in the three following examples. In the settings of the optimization problem, we consider a free final-time $t_{f}$ and we set a timediscretization of 130 steps. We choose a target $\mathcal{T}_{\epsilon}$ with a contamination coefficient $\epsilon=0.1$. We consider that the upper-bound on the control is $D_{\max }=1.5$, dimensioned right above the maximum growth rate of microalgae (see Figure 1). 
Table 1: Parameters of the numerical example.

\begin{tabular}{ccc}
\hline$i$ & $\mu_{i \infty}(L /$ day $)$ & $k_{q i}\left(\mu \mathrm{mol} / \mu m^{3}\right)$ \\
\hline 1 & 1.75 & 1.5 \\
2 & 2 & 1.8 \\
\hline$i$ & $\rho_{m i}\left(\mu m o l / \mu m^{3} /\right.$ day $)$ & $K_{s i}(\mu m o l / L)$ \\
\hline 1 & 9.2 & 0.15 \\
2 & 9.6 & 0.25 \\
\hline \multicolumn{3}{c}{$s_{i n}(\mu m o l / L)$} \\
\hline
\end{tabular}
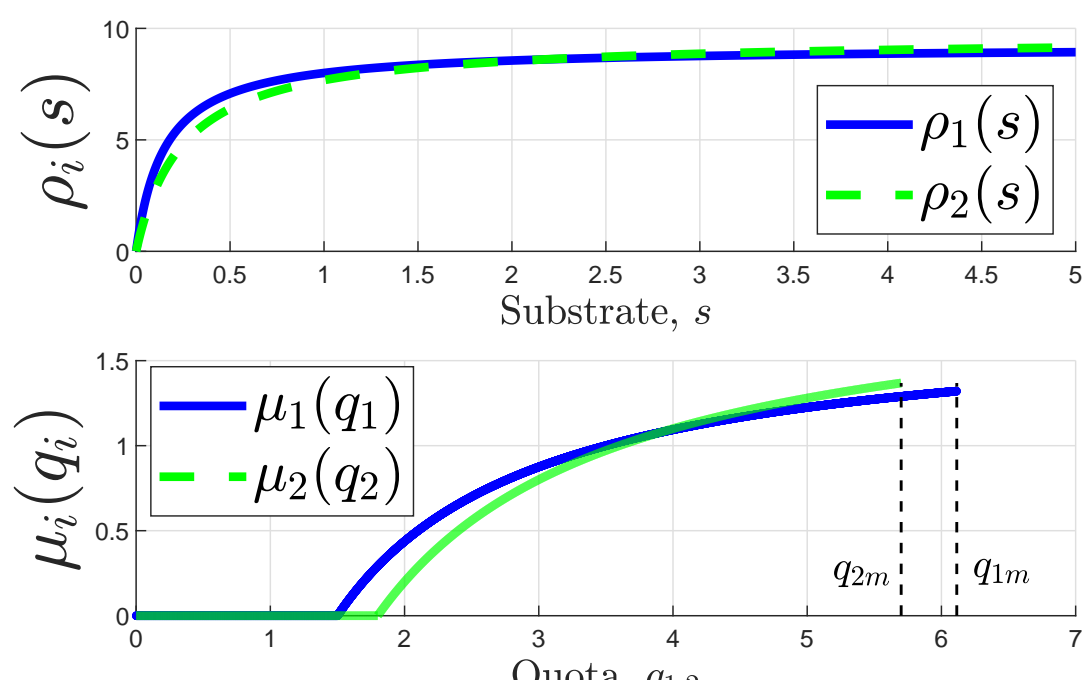

Quota, $q_{1,2}$
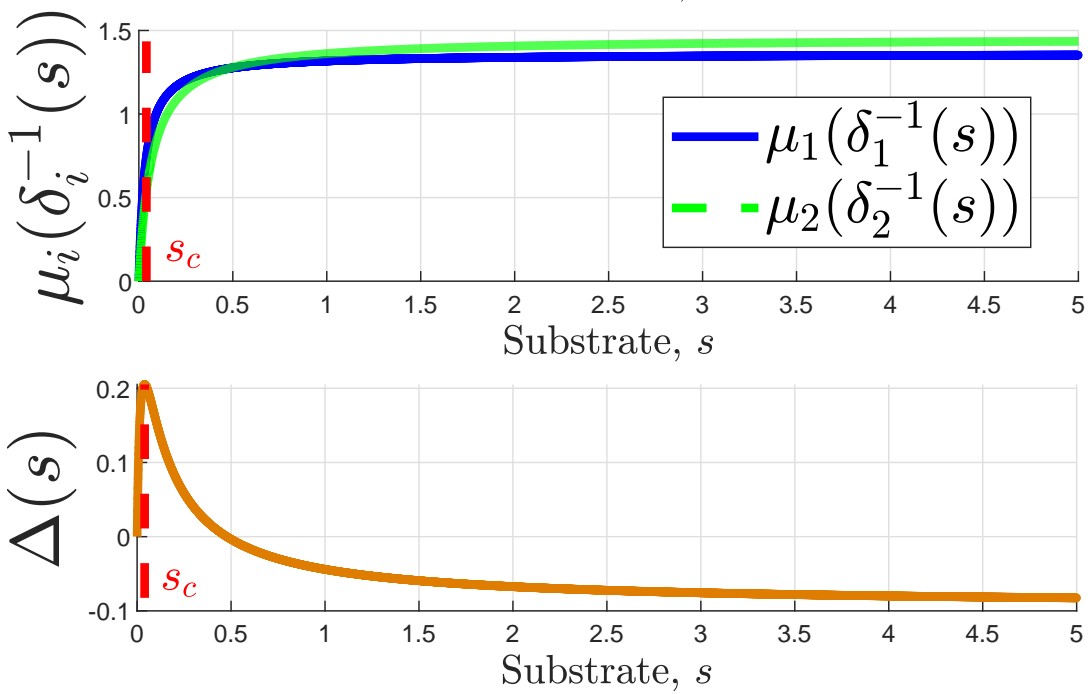

Figure 1: The basic functions corresponding to the parameters in Tab. 1. In this case, we get $s_{c}=0.04$. 
Example 1: We consider here the initial conditions, associated to the model parameters in Table 1 , given by, $s^{0}=2.8 \mu \mathrm{mol} / L, q_{1}^{0}=1.6 \mu \mathrm{mol} / \mu \mathrm{m}^{3}, x_{1}^{0}=0.1 \mu \mathrm{m}^{3} / L, q_{2}^{0}=1.9$ $\mu \mathrm{mol} / \mu \mathrm{m}^{3}, x_{2}^{0}=0.1 \mu \mathrm{m}^{3} / \mathrm{L}$. We note that these initial conditions do not belong to the positively invariant set $\mathcal{F}$ and that they correspond to the case $\mathbf{1}$.

The minimal-time required to reach the target $\mathcal{T}_{\epsilon}$ is $t_{f}=13.0151$ days, after which we get $x_{1}\left(t_{f}\right)=1.9366 \mu \mathrm{m}^{3} / L$ and $x_{2}\left(t_{f}\right)=0.1936 \mu \mathrm{m}^{3} / L$.

The optimal control provided by Bocop is given in Figure 2-(a). The structure bang(0)singular of the control is validated by checking that the functions $\Phi$ and $\dot{\Phi}$ are zero. In Figure 2-(b), we observe that $\Delta(s(t))$ evolves sub-optimally on the singular arc for all $t \in\left[t_{s}, t_{f}\right]$, i.e., it evolves around the maximum of the function $\Delta$. The corresponding model trajectories are given in Figure 3. The turnpike behaviors of the trajectories $s, q_{1}$ and $q_{2}$ are illustrated in Figure 3-(b). From the static-OCP (9) discussed in Sect. 4, we obtain using the numerical values in Table 1 and in Figure 1, the optimal steady-state,

$$
\mathcal{E}=\left(s_{c}, q_{1 c}, q_{2 c}\right), \quad \text { where }, s_{c}=0.04, \quad q_{1 c}=2.6068, \quad q_{2 c}=2.4621
$$

In Figure 4 the co-state trajectories are illustrated. The numerical analysis reveals that the switching time instant $t_{s}$ is characterized by the fact that the co-state $\lambda_{s}$ vanishes at that time, as illustrated in Figure 4-(b), from which we determine that $t_{s}=1.0928$ days. Moreover, the co-states $\lambda_{s}, \lambda_{q_{1}}$ and $\lambda_{q_{2}}$ show a turnpike property, as illustrated in Figure 4-(b), i.e. $\lambda_{s}, \lambda_{q_{1}}$ and $\lambda_{q_{2}}$ tend to be close to the static-optimal solution based on $s_{c}$.

Next, in Figure 3-(b), we note that the substrate $s$ is not constant on the singular arc, but it remains in the neighborhood of $s_{c}$.

Finally, we see in Figure 5 that the time required by the five trajectories to converge towards the positively invariant set $\mathcal{F}$ is not negligible compared to final-time $t_{f}$. The ratio $x_{1}\left(t_{f}\right) / x_{2}\left(t_{f}\right)$ with optimal $D$ is compared to the case when $D$ is constant $(D=0.1$ and $D=1.2$ ), showing the interest of the analysis.
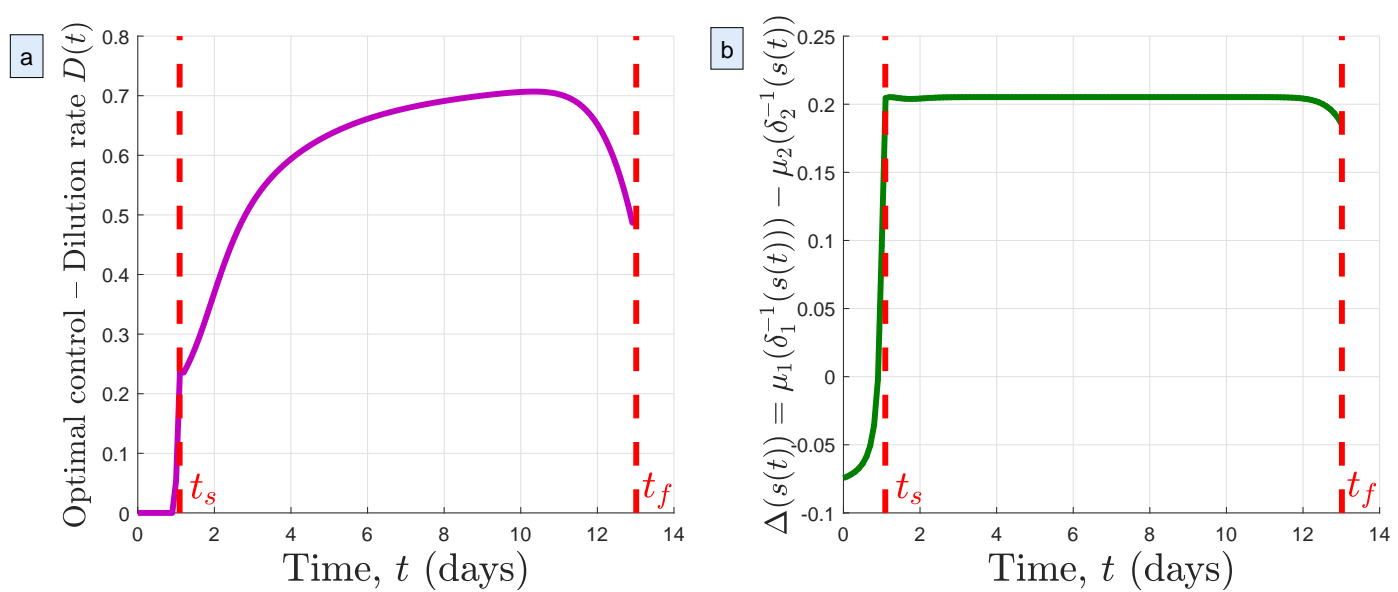

Figure 2: (a) The optimal control provided by Bocop when $s^{0}=1>s_{c}$ is a bang(0)-singular control law. (b) The function $\Delta(s(t))$ for all $t \in\left[0, t_{f}\right]$. 

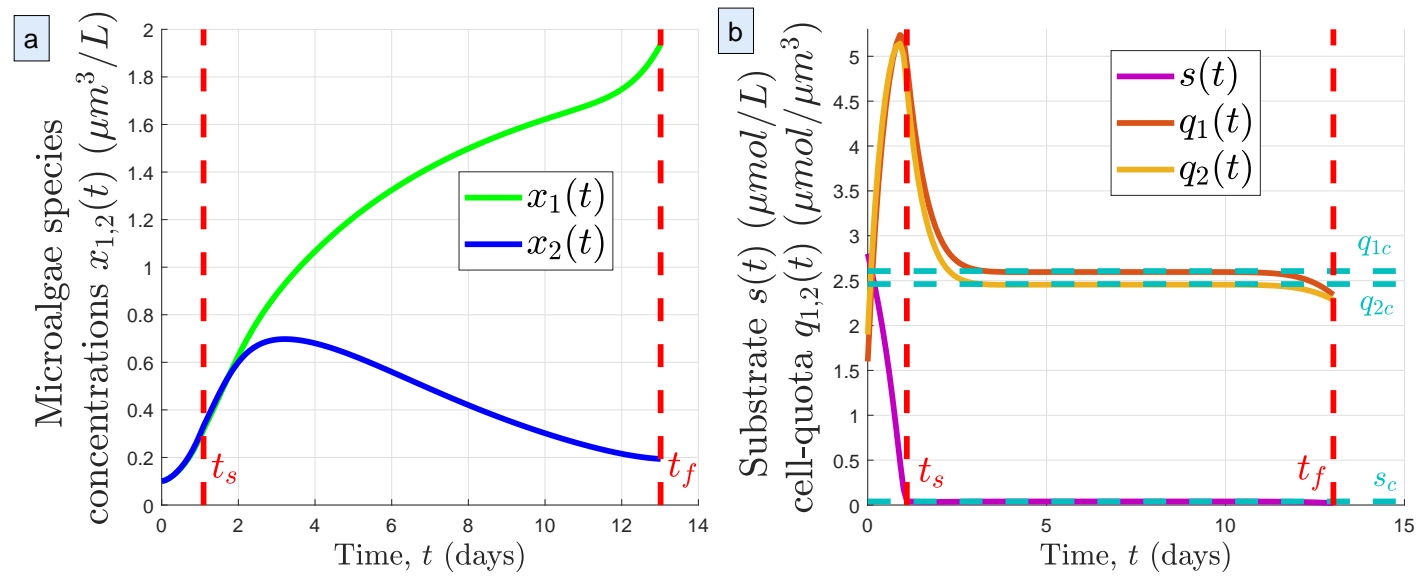

Figure 3: Trajectories associated to the initial conditions in Example 1, in closed loop with the bang(0)singular control law in Figure 2. (a) The ratio $x_{1} / x_{2}=0.1$ is reached at $t_{f}=13.0151$ days. (b) Turnpike features of the optimal trajectories $s, q_{1}$ and $q_{2}$ in Example 1.
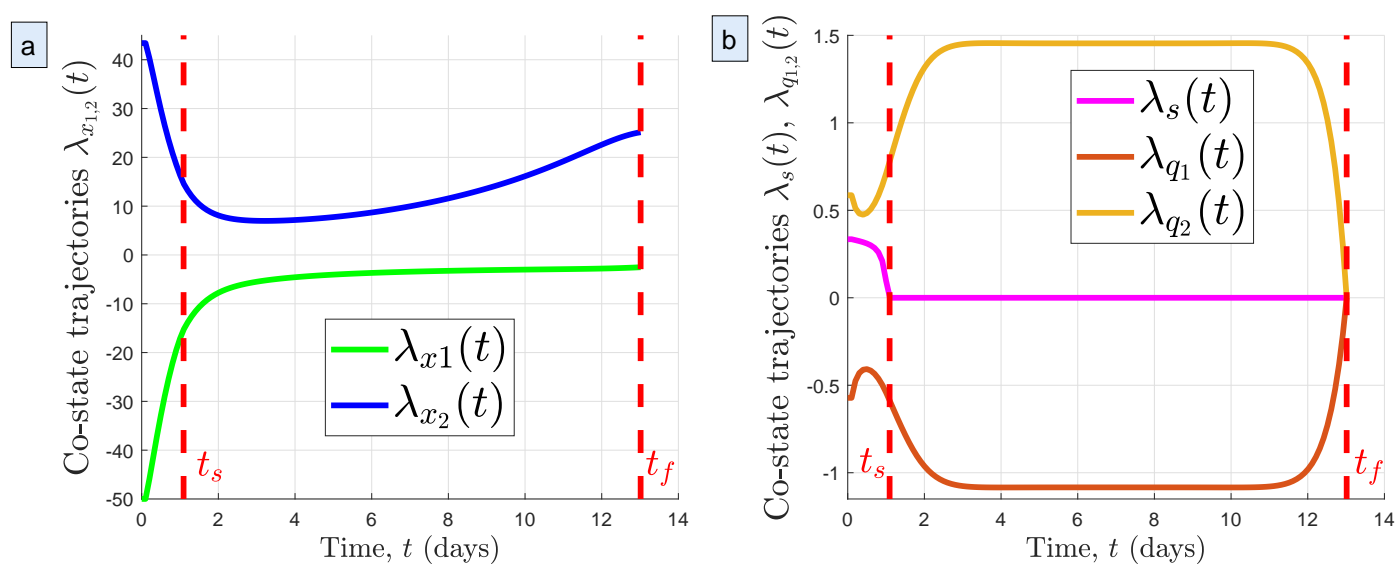

Figure 4: (a) Co-state trajectories $\forall t \in\left[0, t_{f}\right]$ of $x_{1}$ and $x_{2}$ in Example 1. (b) Co-state trajectories $\forall t \in\left[0, t_{f}\right]$ of $s, q_{1}$ and $q_{2}$ in Example 1. The switching-time $t_{s}$ corresponds to the instant at which $\lambda_{s}=0$. The co-state of the $s$-dynamics remains zero until reaching the target $\mathcal{T}_{\epsilon}$.

Example 2: Now we illustrate the case 2 characterized by $s^{0}<s_{c}$. More precisely, we consider the initial conditions outside $\mathcal{F}$ given by, $s^{0}=0.01 \mu \mathrm{mol} / L, q_{1}^{0}=1.6 \mu \mathrm{mol} / \mathrm{\mu m}^{3}$, $x_{1}^{0}=2.5 \mu \mathrm{m}^{3} / L, q_{2}^{0}=1.85 \mu \mathrm{mol} / \mu \mathrm{m}^{3}, x_{2}^{0}=2.5 \mu \mathrm{m}^{3} / L$. The minimal-time required to reach the target is given by $t_{f}=11.5434$ days, after which we end up with $x_{1}\left(t_{f}\right)=1.9374$ $\mu \mathrm{m}^{3} / L$ and $x_{2}\left(t_{f}\right)=0.1937 \mu \mathrm{m}^{3} / L$. The optimal control in this case is of type bang-singular, where the bang is $D_{\max }=1.5$, as illustrated in Figure 6-(a). The evolution of $\Delta(s(t))$ for all $t \in\left[0, t_{f}\right]$ is given in Figure 6-(b). It evolves around the maximum of the function $\Delta$ for almost all $t \in\left[t_{s}, t_{f}\right]$. The optimal trajectories of the studied system, in closed loop with the optimal control, are illustrated in Figure 7-(a). A turnpike property also holds for the trajectories $s, q_{1}$ and $q_{2}$, as well as their co-states (Figure 8-(a)). As previously mentioned, the switching instant $t_{s}$ is identified numerically from the co-state of the substrate $s$. As indicated in Figure 8-(a), we get, $t_{s}=0.4405$ days. Finally, as in Example 1, we check in Figure 8-(b) the convergence rate to the positively invariant set $\mathcal{F}$. 

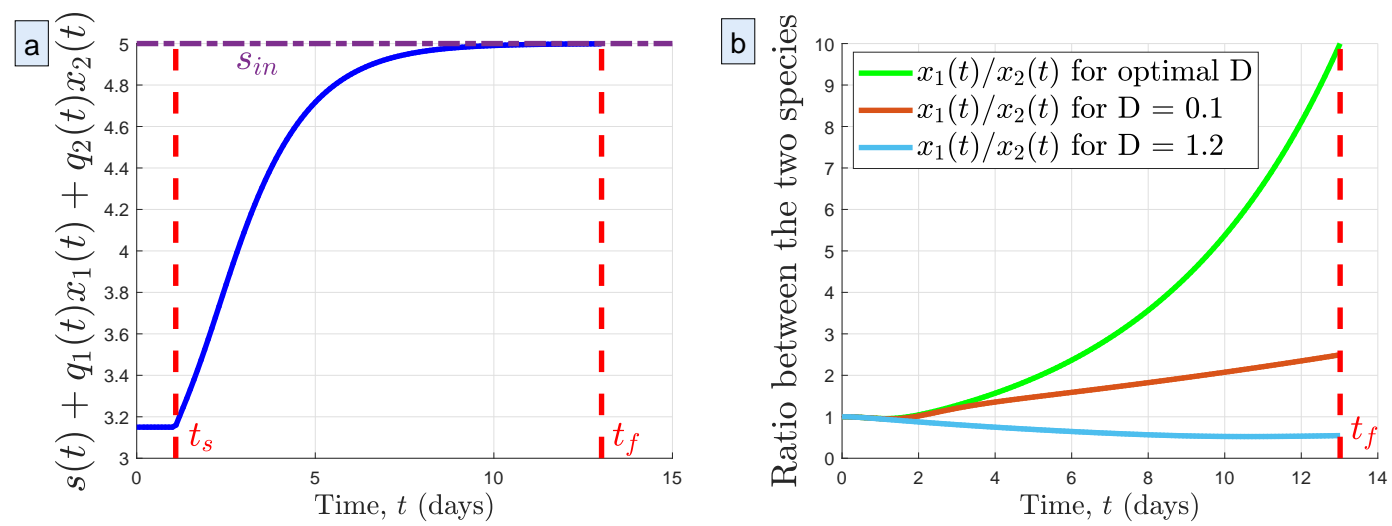

Figure 5: (a) Convergence to the positively invariant set $\mathcal{F}$ in Example 1. (b) Assessment of the optimal strategy: evolution of the ratio $x_{1} / x_{2}$ with the optimal control compared to a fixed constant $D$.
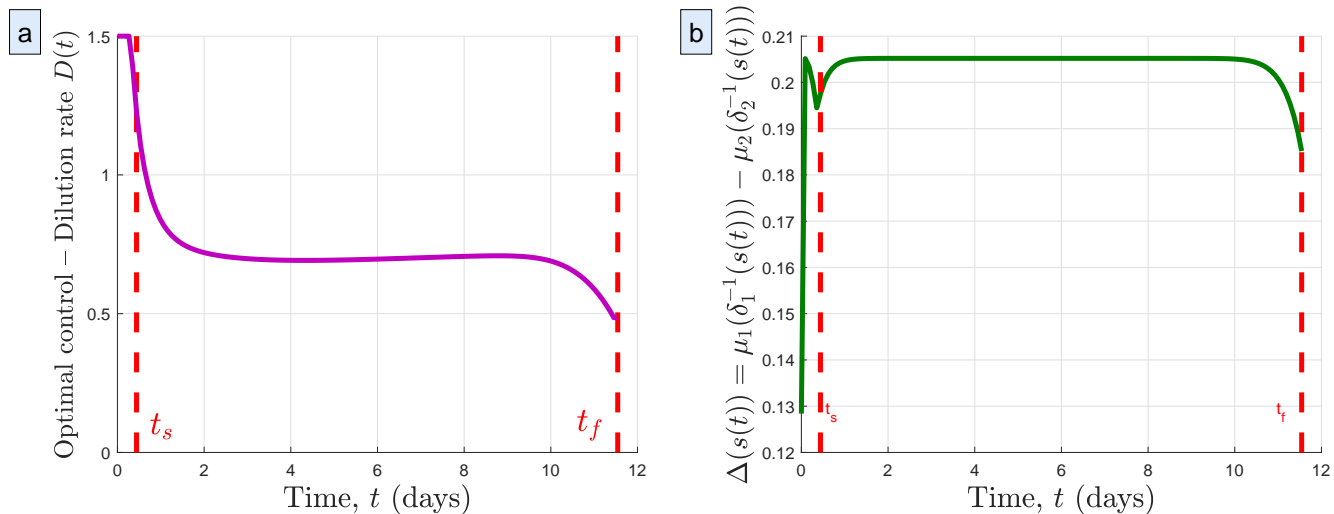

Figure 6: (a) The optimal control in the case where $s^{0}<s_{c}$ is of type bang $\left(D_{\max }\right)$-singular in Example 2. (b) The function $\Delta(s(t))$ for all $t \in\left[0, t_{f}\right]$ in Example 2 .
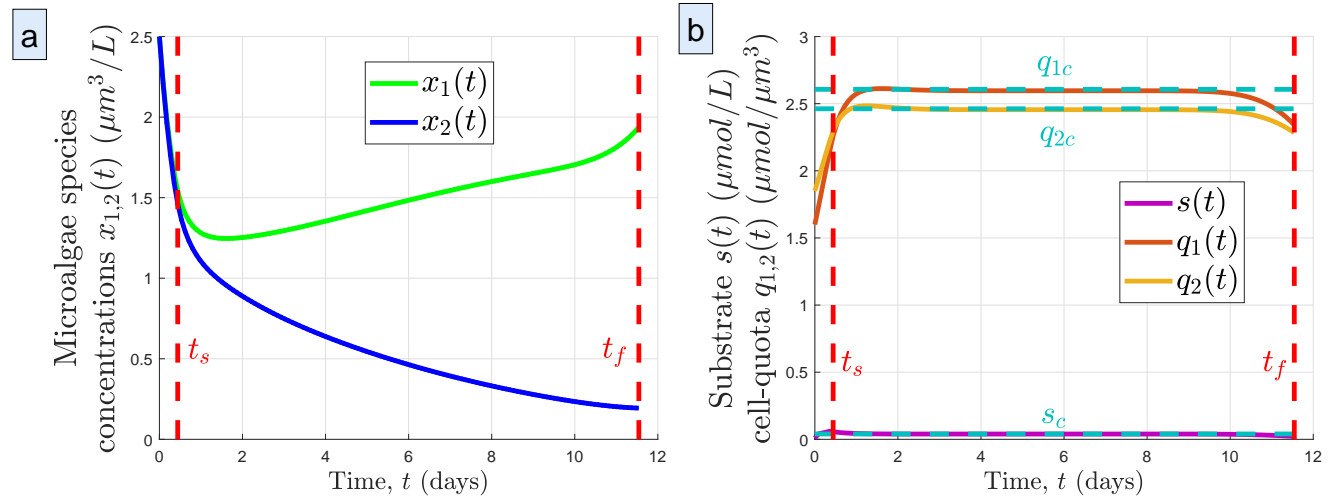

Figure 7: Trajectories associated to the initial conditions in Example 2, in closed loop with the $\operatorname{bang}\left(D_{\max }\right)$ singular control law in Figure 6. (a) The ratio $x_{1}(t) / x_{2}(t)=0.1$ is reached at $t_{f}=11.5434$ days. (b) Turnpike features of the optimal trajectories $s, q_{1}$ and $q_{2}$ in Example 2. 

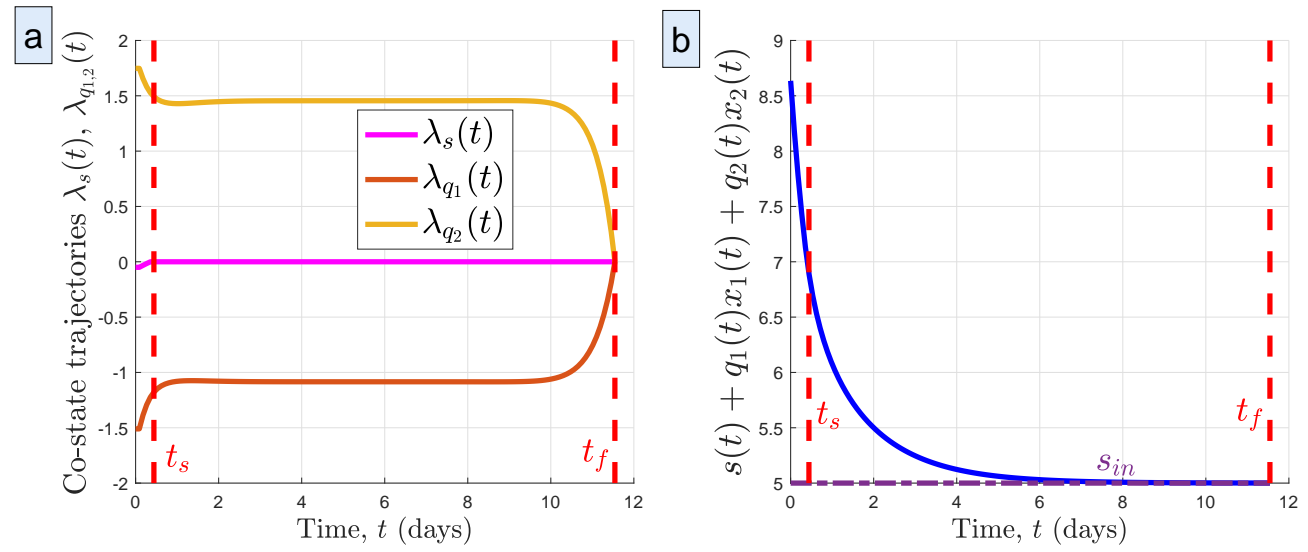

Figure 8: (a) Co-state trajectories $\forall t \in\left[0, t_{f}\right]$ of $s, q_{1}$ and $q_{2}$ in Example 2, showing a turnpike-type feature. The switching-time $t_{s}$ corresponds to the instant at which $\lambda_{s}=0$. The co-state of the $s$-dynamics remains zero until reaching the target $\mathcal{T}_{\epsilon}$. (b) Convergence to the positively invariant set $\mathcal{F}$.

Example 3: (Selection of the second species). Extending the arguments for the selection of the first species, we can deduce that the optimal selection of the second species is related to the maximization of $\Delta(s)=\mu_{2}\left(\delta_{2}^{-1}(s)\right)-\mu_{1}\left(\delta_{1}^{-1}(s)\right)$. Pay attention that now species 2 is the winner.

For the model parameters in Table 1 and Figure 1, we can see that the function $\Delta(s)=$ $\mu_{2}\left(\delta_{2}^{-1}(s)\right)-\mu_{1}\left(\delta_{1}^{-1}(s)\right)$ has its maximum at $s_{i n}$ which means that the corresponding $s_{c}$ is $s_{i n}$. Therefore, it is expected that the optimal control $D$ aims to steer the substrate $s$ from its initial value $s^{0}$ in the direction of $s_{i n}$. For that purpose, the optimal control is $D=D_{\max }$, driving $s^{0}$ towards $s_{c}=s_{i n}$ in the fastest way.

We perform a numerical simulation on Bocop using the initial conditions, $s^{0}=4 \mu \mathrm{mol} / \mathrm{L}$, $q_{1}^{0}=1.6 \mu \mathrm{mol} / \mu \mathrm{m}^{3}, x_{1}^{0}=0.7 \mu \mathrm{m}^{3} / L, q_{2}^{0}=2 \mu \mathrm{mol} / \mu \mathrm{m}^{3}, x_{2}^{0}=0.7 \mu \mathrm{m}^{3} / L$, with the same contamination rate $\epsilon=0.1$ on the new target $\left(\epsilon x_{2}\left(t_{f}\right)<x_{1}\left(t_{f}\right)\right)$. As expected, the optimal control that achieves minimal-time separation is $D_{\max }=1.5$ as illustrated in Figure 9-(a). A short singular arc appears at the end of the control strategy, that corresponds to $\Phi(t)=0$, $t \in\left[t_{s}, t_{f}\right]$, as illustrated in Figure 9-(b).

Figure 10 illustrates the model trajectories in closed loop with the optimal control given in Figure 9-(a). In this example, simple calculation allow us to solve the static-OCP (9), considering the new form $\mu_{2}\left(q_{2}\right)-\mu_{1}\left(q_{1}\right)$. Thus, we determine the optimal steady-state,

$$
\mathcal{E}=\left(s_{c}, q_{1 c}, q_{2 c}\right), \quad \text { where, } s_{c}=s_{i n}=5, \quad q_{1 c}=6.6040, \quad q_{2 c}=6.3714 .
$$

A turnpike-like feature appears in Figure 10-(b). On the short-time singular arc activated on $\left[t_{s}, t_{f}\right]$, the co-states $\lambda_{s}, \lambda_{q_{1}}$, and $\lambda_{q_{2}}$ converge to the terminal transversality conditions (Figure 11-(a)). We notice in this example that the turnpike-like feature appears in the state trajectories and also in their corresponding co-states (Figure 11-(a)) where the states are most of the time around the optimal steady-state $\mathcal{E}$. Finally, convergence towards the positively invariant set $\mathcal{F}$ is illustrated in Figure 11-(b). 

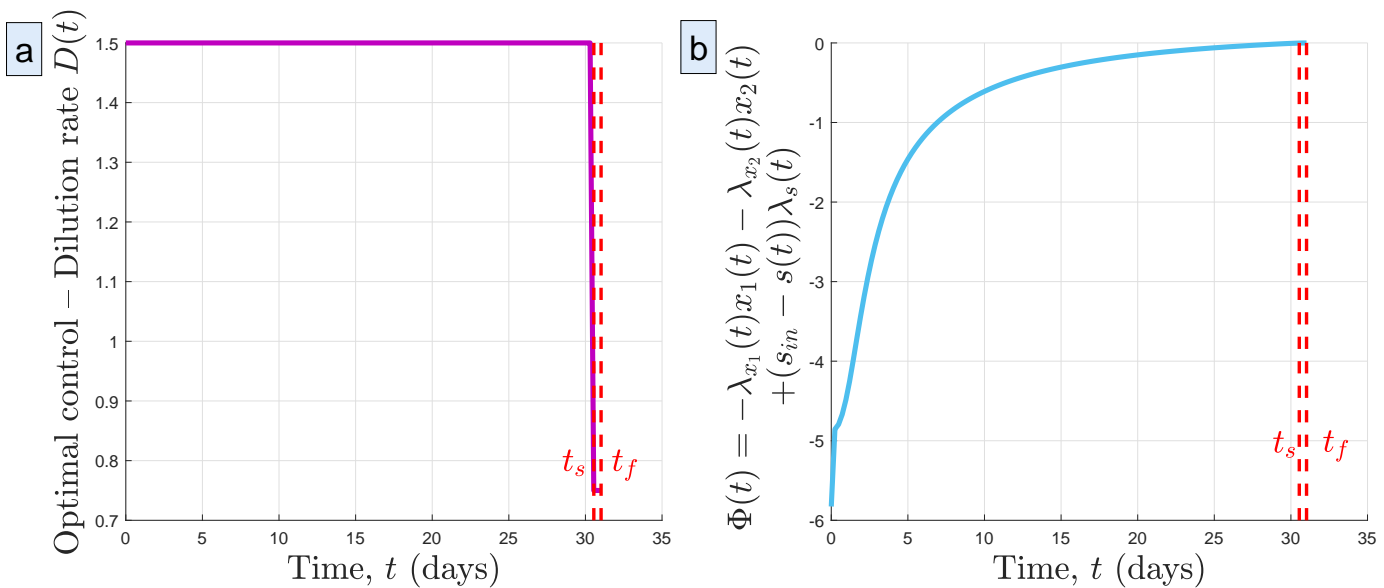

Figure 9: (a) The optimal control in Example 3. (b) Evolution of $\Phi(t)$ for all $t \in\left[0, t_{f}\right]$ in Example 3.
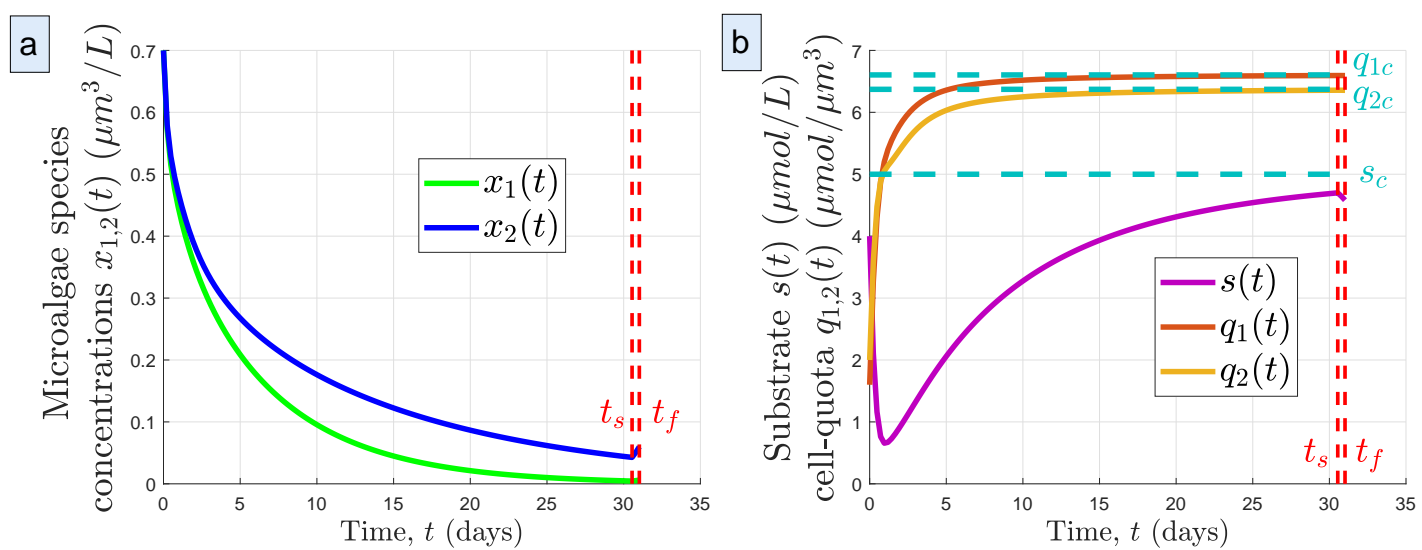

Figure 10: Trajectories associated to the initial conditions in Example 3, in closed loop with the control law in Figure 9-(a). (a) The biomass concentrations $x_{1}(t)$ and $x_{2}(t)$ for all $t \in\left[0, t_{f}\right]$. (b) Turnpike features of the optimal trajectories $q_{1}$ and $q_{2}$ in Example 3 .

\section{Conclusion}

We have investigated the issue of minimal-time selection of microalgae in a photobioreactor governed by the Droop's model. From the insights given by Pontryagin's principle, and using a direct method for the optimization problem, we show that the optimal feedback law is of type bang-singular, where the bangs are of two types ( 0 and $\left.D_{\text {max }}\right)$, depending on the substrate initial state. We have also highlighted a turnpike feature that appears in the optimal separation strategy. It is worth noting that this optimal strategy shares some common features with the one determined for Monod-driven species, but the strategy for the Droop's model is more complex. The geometrical arguments from [1] do not hold anymore here mainly due to the higher state dimension for the Droop case. Thus, other approaches are still required to prove Conjecture 1 for these more complex system dynamics. 

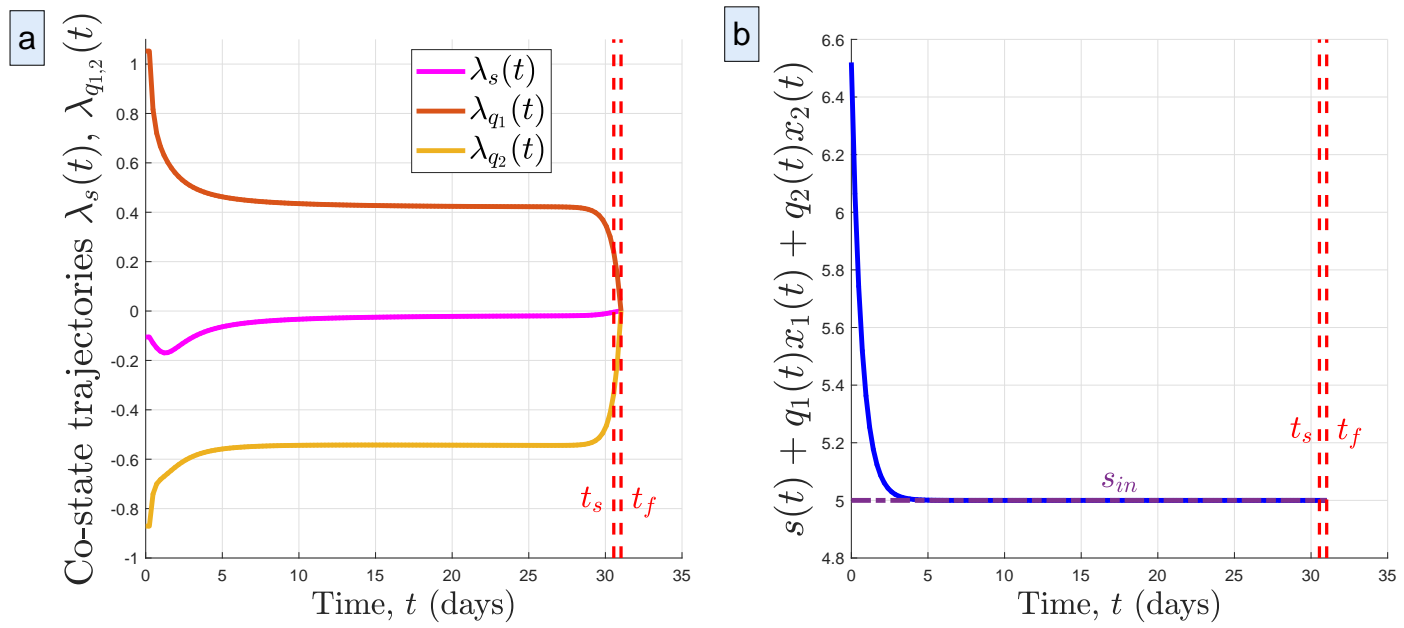

Figure 11: (a) Co-state trajectories $\forall t \in\left[0, t_{f}\right]$ of $s, q_{1}$ and $q_{2}$ in Example 3, showing a turnpike-type feature. (b) Convergence to the positively invariant set $\mathcal{F}$.

\section{Acknowledgements}

This work was partially supported by the Inria Project Lab (IPL) Algae in silico and the project Bio-MSA, Graine-ADEME (France).

\section{References}

[1] T. Bayen, \& F. Mairet, Optimization of the separation of two species in a chemostat. Automatica, 50(4), pp. 1243-1248, (2014).

[2] T. Bayen, \& F. Mairet, Optimization of strain selection in evolutionary continuous culture. International Journal of Control, 90(12), pp. 2748-2759, (2017).

[3] O. Bernard, \& J.L. Gouzé, Transient behavior of biological loop models with application to the Droop model., Mathematical Biosciences, 127(1), pp.19-43., (1995).

[4] O. Bernard, F. Mairet, B. Chachuat, Modelling of microalgae culture systems with applications to control and optimization. Microalgae Biotechnology. Springer, Cham, pp. 59-87, (2015).

[5] O. Bernard, Hurdles and challenges for modelling and control of microalgae for CO2 mitigation and biofuel production. Journal of Process Control 21, no. 10, pp. 1378-1389, (2011).

[6] J. T. Betts, Practical methods for optimal control and estimation using nonlinear programming. Siam, Advances in Design \& Control, 2nd Edition, Vol. 19, p. 427, (2010).

[7] L. T. Biegler, Nonlinear Programming: Concepts, Algorithms, and Applications to Chemical Processes. MPS-SIAM Series on Optimization (Book 10), SIAM-Society for Industrial and Applied Mathematics, p. $415,(2010)$.

[8] F. J. Bonnans, D. Giorgi, V. Grelard, B. Heymann, S. Maindrault, P. Martinon, O. Tissot, J. Liu, BOCOP: an open source toolbox for optimal control - A collection of examples. Team Commands, Inria Saclay, Technical Reports, http://bocop.org, (2017).

[9] H. Bonnefond, G. Grimaud, J. Rumin, G. Bougaran, A. Talec, M. Gachelin, M. Boutoute, E. Pruvost, O. Bernard, A. Sciandra, Continuous selection pressure to improve temperature acclimation of Tisochrysis lutea. PloS one, Num. 9, Vol. 12: e0183547, Public Library of Science, (2017).

[10] J. Caperon, \& J. Meyer, Nitrogen-limited growth of marine phytoplankton-II. Uptake kinetics and their role in nutrient limited growth of phytoplankton. In Deep Sea Research and Oceanographic Abstracts, Vol. 19, No. 9, pp. 619-632, (1972). 
[11] W. Djema, L. Giraldi, O. Bernard, An Optimal Control Strategy Separating Two Species of Microalgae in Photobioreactors., Conference on Dynamics and Control of Process Systems, including Biosystems,12th DYCOPS, IFAC PapersOnLine 52-1, pp.910-915, (2019). https://doi.org/10.1016/j.ifacol.2019.06.178

[12] M. R. Droop, Vitamin $B_{12}$ and marine ecology. IV. The kinetics of uptake growth and inhibition in Monochrysis lutheri. J. Mar. Biol. Assoc. 48 (3), pp. 689-733, (1968).

[13] M. R. Droop, Some thoughts on nutrient limitation in algae. Journal of Phycology, 9(3), pp.264-272, (1973).

[14] M. R. Droop, 25 years of algal growth kinetics, a personal view. Bot. Mar. 16, pp. 99-112, (1983).

[15] F. Grognard, P. Masci, E. Benoît, O. Bernard, Competition between phytoplankton and bacteria: exclusion and coexistence. J. of Math. Bio., 70(5), pp. 959-1006, (2015).

[16] E. Hairer, S.P. Norsett, G. Wanner, Solving ordinary differential equations., 1, Nonstiff problems. Springer-Vlg., (1991).

[17] L. M. Hocking, Optimal control: an introduction to the theory with applications. Oxford University Press, (1991).

[18] S. B. Hsu, \& T. H. Hsu, Competitive exclusion of microbial species for a single nutrient with internal storage. SIAM J. on Applied Math., 68(6), pp.1600-1617, (2008).

[19] S. B. Hsu, S. Hubbell, P. Waltman, A mathematical theory for single-nutrient competition in continuous cultures of micro-organisms. SIAM Journal on Applied Mathematics, Vol. 32, No. 2, pp. 366-383, (1977).

[20] S. Liu, Bioprocess Engineering: Kinetics, Sustainability, and Reactor Design. 2nd Ed., Elsevier, p. 1172, (2016).

[21] P. Masci, O. Bernard, F. Grognard, Continuous selection of the fastest growing species in the chemostat., IFAC World Congress, IFAC Proceedings, 41(2), pp.9707-9712, (2008).

[22] J. Monod, Recherches sur la croissance des cultures bactériennes., Paris: Herman., (1942).

[23] J. Monod, La technique de culture continue; théorie et applications. Annales de l'Institute Pasteur 79: pp. 390-401, (1950).

[24] L. S. Pontryagin, V. G. Boltyanskiy, R. V. Gamkrelidze, E. F. Mishchenko, Mathematical theory of optimal processes. New York, NY Springer, (1964).

[25] H. L. Smith, \& P. Waltman, The theory of the chemostat: dynamics of microbial competition., Cambridge Studies in Mathematical Biology, Cambridge Univ. Press., (1995).

[26] E. Trélat, \& E. Zuazua, The turnpike property in finite-dimensional nonlinear optimal control., Journal of Differential Equations, 258(1), pp.81-114., (2015).

[27] E. Trélat, C. Zhang, Integral and measure-turnpike properties for infinite-dimensional optimal control systems., Mathematics of Control, Signals \& Systems, 30(1), p.3., (2018). 